\title{
Beam-Scanning Reflectarray Based on a Single Varactor-Tuned Element
}

\author{
F. Venneri, S. Costanzo, G. Di Massa, E. Marozzo, A. Borgia, P. Corsonello, and M. Salzano \\ Dipartimento di Elettronica, Informatica e Sistemistica, Università della Calabria, 87036 Rende, Italy \\ Correspondence should be addressed to S. Costanzo, costanzo@deis.unical.it
}

Received 3 February 2012; Accepted 16 April 2012

Academic Editor: Raphaël Gillard

Copyright () 2012 F. Venneri et al. This is an open access article distributed under the Creative Commons Attribution License, which permits unrestricted use, distribution, and reproduction in any medium, provided the original work is properly cited.

A single varactor-tuned element is investigated as effective solution for the realization of reconfigurable reflectarray antennas. The proposed configuration is successfully implemented for the design of an X-band reflectarray prototype of $3 \times 15$ radiators. A dedicated electronic control board is designed as an integral part of the antenna. Good performances in terms of beam-scanning capabilities are obtained from measurements.

\section{Introduction}

Microstrip reflectarray antennas have received considerable interests in the last two decades, due to their appealing features in terms of low cost, high efficiency, and reduced mass and volume. Many different implementations of the reflectarray concept have been proposed, ranging from the first simple configurations, based on the use of variable size patches [1] or identical elements with variable length stubs [2], up to more sophisticated structures, such three layers stacked [3] or aperture coupled patches [4]. The success obtained in the realization of passive reflectarray structures has recently attracted the attention towards the application of this technology to the design of reconfigurable reflector antennas. The main advantage deriving from the development of active reflectarrays over traditional-phased arrays is the elimination of complicated beam-forming networks, which typically introduce very high losses. Many different configurations have been proposed as phase-tunable reflectarray elements such as mechanically reconfigurable patches $[5,6]$, varactor-loaded microstrip radiators [7-9], reflectarrays printed on tunable liquid crystal substrates [10], and MEMS-based structures [11-14].

In this paper, a tunable radiating element based on the use of a single varactor diode [9] is implemented for the realization of a reconfigurable reflectarray antenna. The adopted element consists of a patch aperture-coupled to a varactor loaded line. The phase tuning capabilities of this configuration have been already demonstrated by the authors in [9]. When compared with the existing solutions, the adopted configuration offers a uniform reflecting surface, due to the fact that the tunable element and the biasing circuitries are located on the opposite side below the ground plane. Furthermore, as demonstrated in [9], an optimum phase tuning range of about 320 degrees is obtained by integrating a single diode to each radiating element.

The proposed configuration is properly synthesized for the design of an $X$-band reflectarray with beam-scanning capabilities. A printed electronic circuit is integrated to the antenna in order to actively control the bias voltages across the diodes. The radiation pattern measurements of the reflectarray prototype show the ability to electronically scan the main beam along some prescribed directions.

\section{Reflectarray Element Design}

The single radiator adopted for the realization of the proposed reconfigurable reflectarray is based on the use of a patch printed on a grounded dielectric substrate and slot coupled to a varactor loaded microstrip line (Figure 1). By changing the bias voltage across the diode, a variable capacitive load is obtained, which in turns introduces a tunable phase shift in the reradiated field. A biasing line is printed on the tuning line substrate, thus the reflectarray surface is 


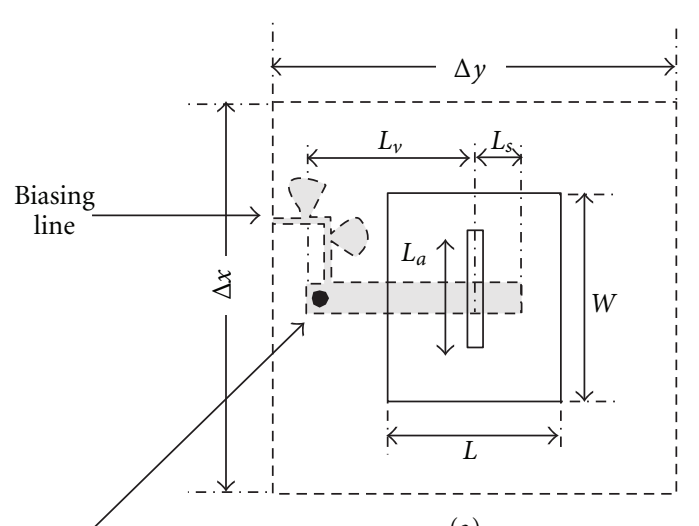

(a)

Varactor

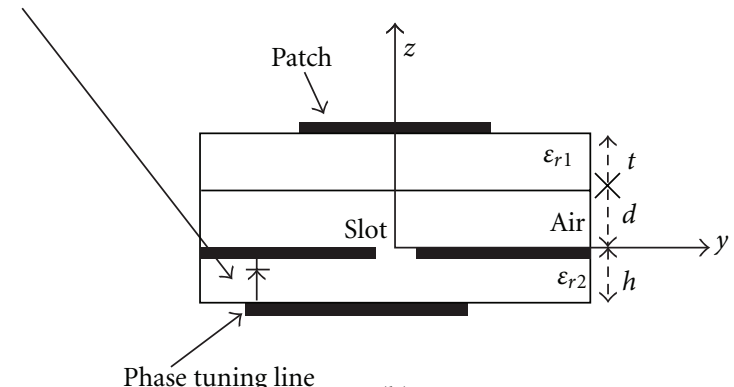

(b)

FIGURE 1: Reflectarray element configuration: (a) top view; (b) side view.

simply composed by identical rectangular patches separated by uniform spacings. This last feature makes the proposed reflectarray configuration free from unwanted reflections.

The adopted reflectarray element is designed in order to obtain a full phase tuning range at the operating frequency $f_{0}=11.5 \mathrm{GHz}$. A MoM-based infinite array approach is adopted for the simulation and the optimization of the radiating structure. A cell size of $\Delta x \times \Delta y=0.7 \lambda_{0} \times 0.7 \lambda_{0}$ is fixed at the operating frequency $f_{0}$. Two identical dielectric slabs are chosen as patch and line substrates, having the following features: $\varepsilon_{r 1}=\varepsilon_{r 2}=2.33, t=h=0.762 \mathrm{~mm}$. A layer of air with a thickness $d=0.762 \mathrm{~mm}$ is placed between the antenna substrate and the ground plane. The following dimensions are obtained for the different parts composing the antenna: $W=9.3 \mathrm{~mm}, L=8.2 \mathrm{~mm}, W_{a}=0.6 \mathrm{~mm}, L_{a}=5.8 \mathrm{~mm}$, $W_{s}=3.07 \mathrm{~mm}$ (Figure 2(a)). The assumed line width $W_{s}$ corresponds to a characteristic impedance of value equal to $40 \Omega$. The input impedance $Z_{t}$, (Figure $2($ a) ), evaluated at the center of the slot, results to be perfectly matched to the $40 \Omega$-line when the stub $L_{s}$ is fixed at a value of $2 \mathrm{~mm}$ (Figure 2(b)).

A Microsemi MV31011-89 varactor diode, with a tunable capacitance ranging from $0.2 \mathrm{pF}\left(V_{\text {bias }}=20\right.$ Volt $)$ to $2 \mathrm{pF}$ $\left(V_{\text {bias }}=0\right.$ Volt $)$, is integrated to the above element in order to obtain the required reconfiguration capabilities. In this case, the two line sections $L_{v}$ and $L_{s}$ (see Figure 1) are optimized in order to maximize the phase agility of the element in the assigned varactor capacitance range. At this purpose, the stub $L_{s}$ is fixed to a value of $7.8 \mathrm{~mm}$, while the length $L_{v}$ is set to

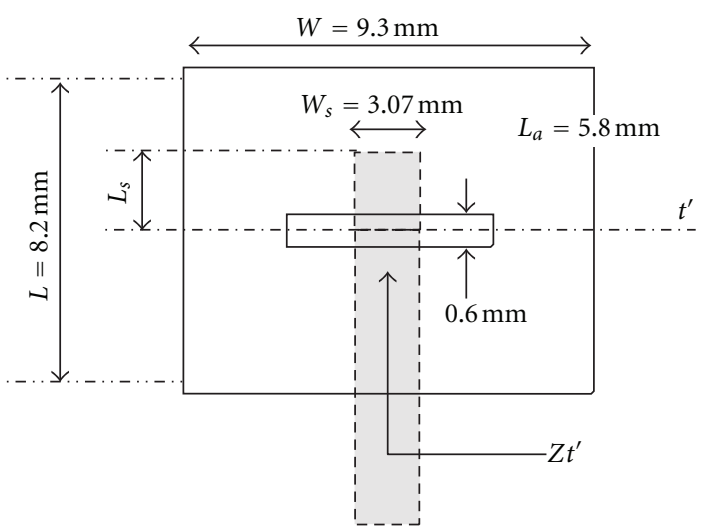

(a)

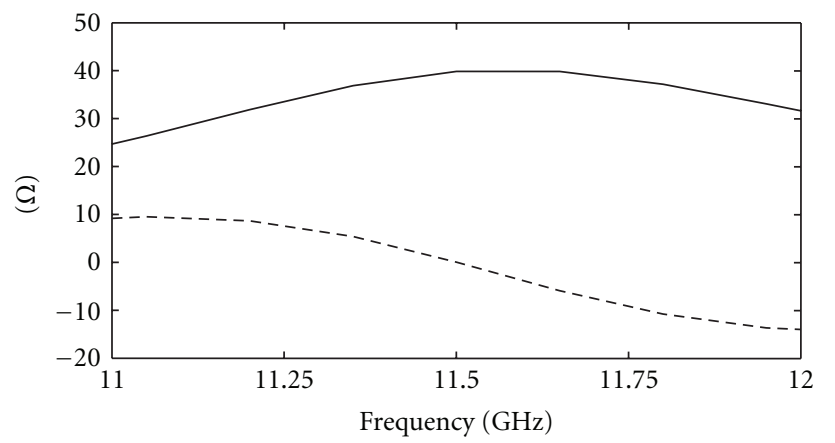

$-\operatorname{Re}\left(Z_{t^{\prime}}\right)-L_{s}=2 \mathrm{~mm}$

$--\operatorname{lm}\left(Z_{t^{\prime}}\right)-L_{s}=2 \mathrm{~mm}$

(b)

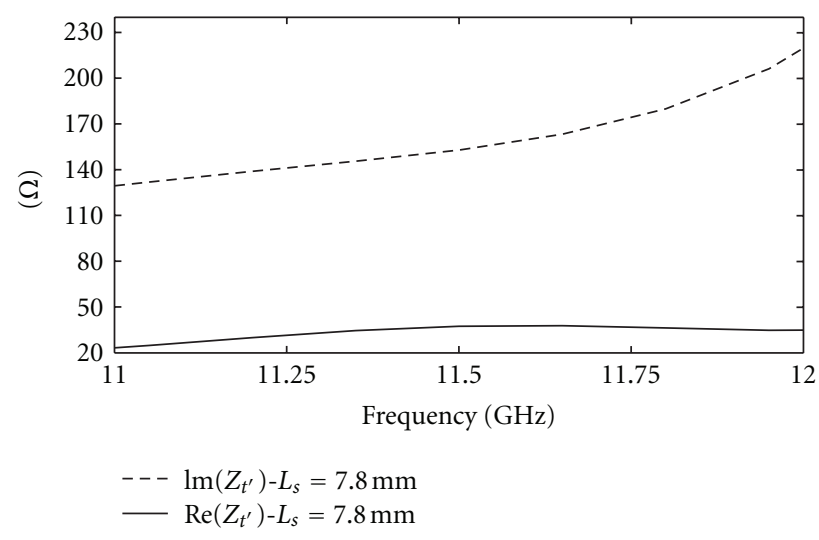

(c)

FIGURE 2: Synthesized aperture-coupled patch: (a) element layout; (b) simulated input impedance for $L_{s}=2 \mathrm{~mm}$; (c) simulated input impedance for $L_{s}=7.8 \mathrm{~mm}$.

the value of $6.5 \mathrm{~mm}$, in order to move the maximum phase variation within the diode capacitance range.

The reflectarray element is analyzed in order to evaluate the behavior of the reflection phase versus diode capacitance, for different values of the stub length. A normally incident plane wave is assumed.

The reflection phase curves, computed in the case of length $L_{s}$ equal to $2 \mathrm{~mm}$ and $7.8 \mathrm{~mm}$, are reported in 


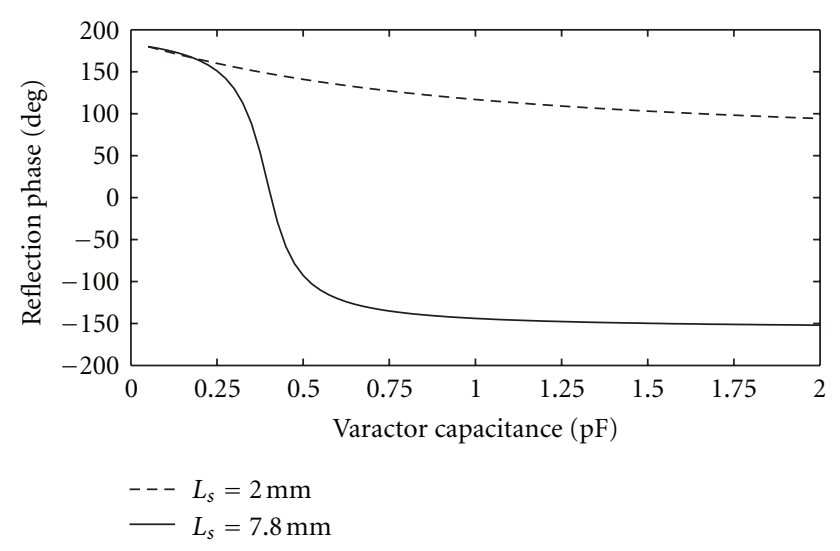

(a)

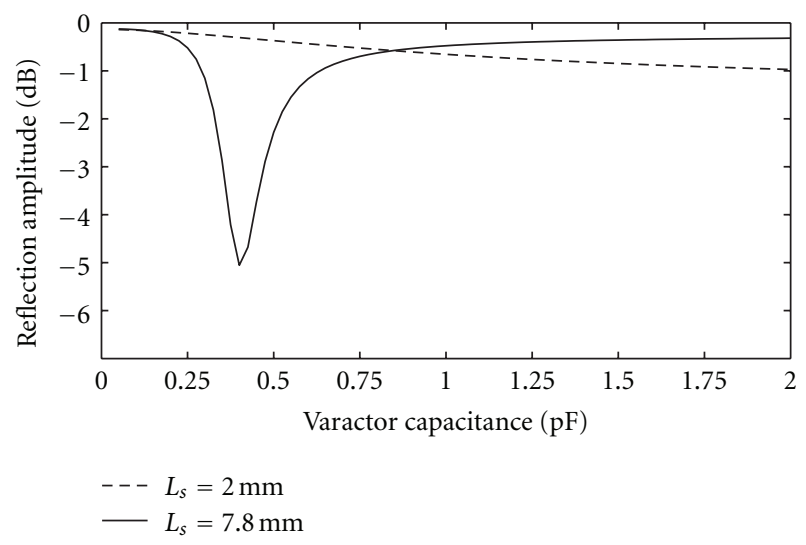

(b)

FIGURE 3: Simulated reflection coefficient for a normally incident plane wave: phase (a) and amplitude (b) versus varactor capacitance for different stub lengths.

Figure 3(a). The curves are computed by varying the diode capacitance $C_{v}$ in the range between $0.2 \mathrm{pF}$ and $2 \mathrm{pF}$. From a comparison of the two considered curves, it can be stated that an higher phase tuning range can be obtained by introducing a proper inductive effect in the input impedance $Z_{t}$, (Figure 2(c)). In this case, more pronounced reflection losses are obtained (Figure 3(b)), which can be reduced by adopting a varactor with a lower series resistance. In the above simulations, a value of $1.36 \Omega$ is assigned for this diode parameter, resulting into a value of $3.7 \mathrm{~dB}$ for the losses due to the varactor insertion.

The correctness of the simulated design curves has been experimentally verified in [9], where a good agreement between measurements and simulations can be appreciated.

\section{Reflectarray Prototype Description and Experimental Results}

The synthesized element is adopted as elementary cell of a $3 \times$ 15 reflectarray (Figure 4(a)). The array is illuminated by an $X$-band horn, located at a distance of $34 \mathrm{~cm}$ from the reflecting surface, with an offset angle of 15 degrees in the $y$ - $z$ plane

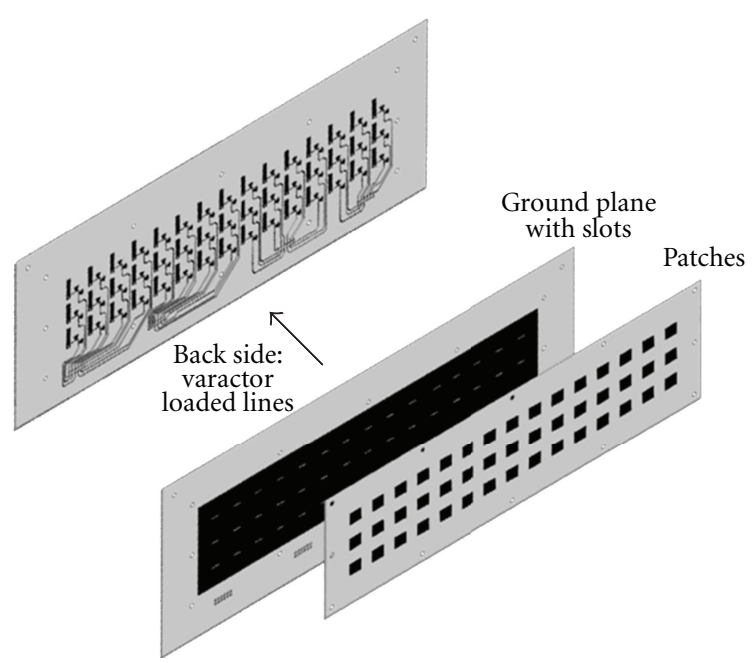

(a)

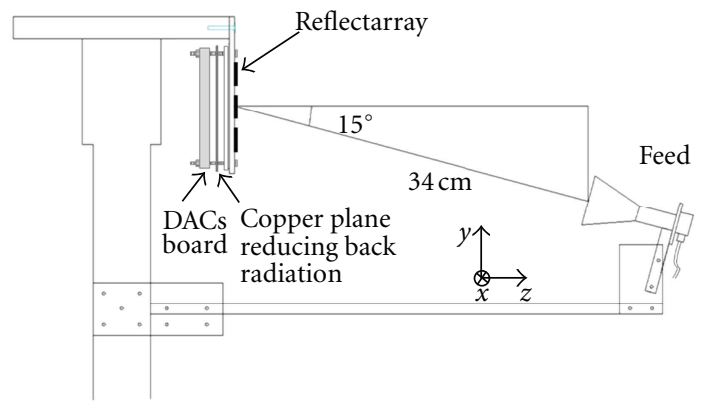

(b)

Figure 4: Reflectarray structure scheme-(a) aperture-coupled array; (b) array illuminated by the feed.

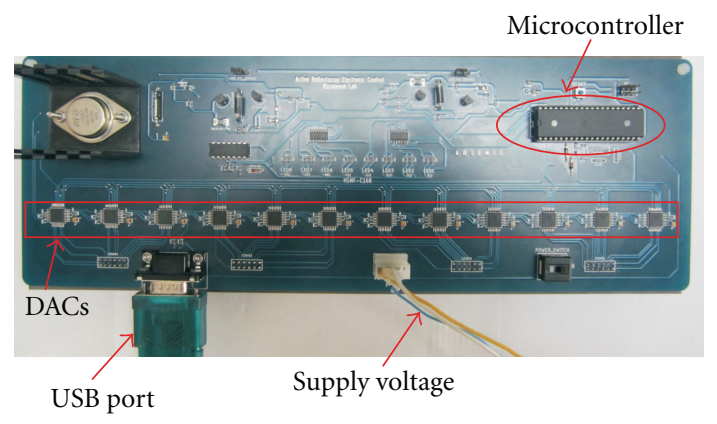

Figure 5: Photograph of the designed DACs board.

(Figure 4(b)). The antenna is integrated with an electronic board composed by an array of digital to analog converters (AD5764R DACs) driven by a microcontroller (ATMEL ATmega1284P). Each AD5764R integrated circuit is composed by four 16-bit DACs. A photograph of the designed electronic board is illustrated in Figure 5 .

A metallic plane is placed between the antenna and the DACs-board (Figure 4(b)) in order to reduce the back-radiation as well as the occurrence of any undesired interference effect. 


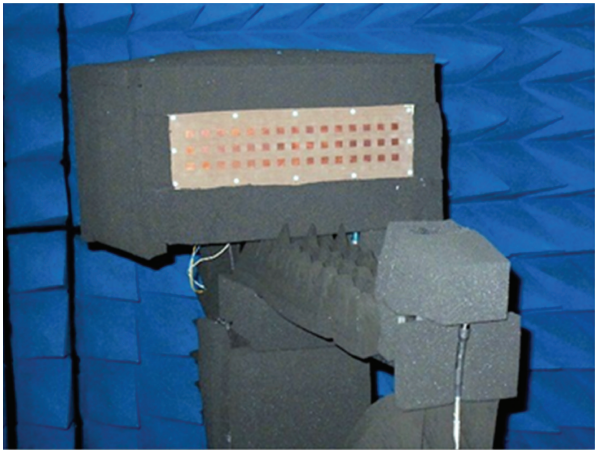

FIGURE 6: Photograph of the reflectarray prototype into the anechoic chamber.

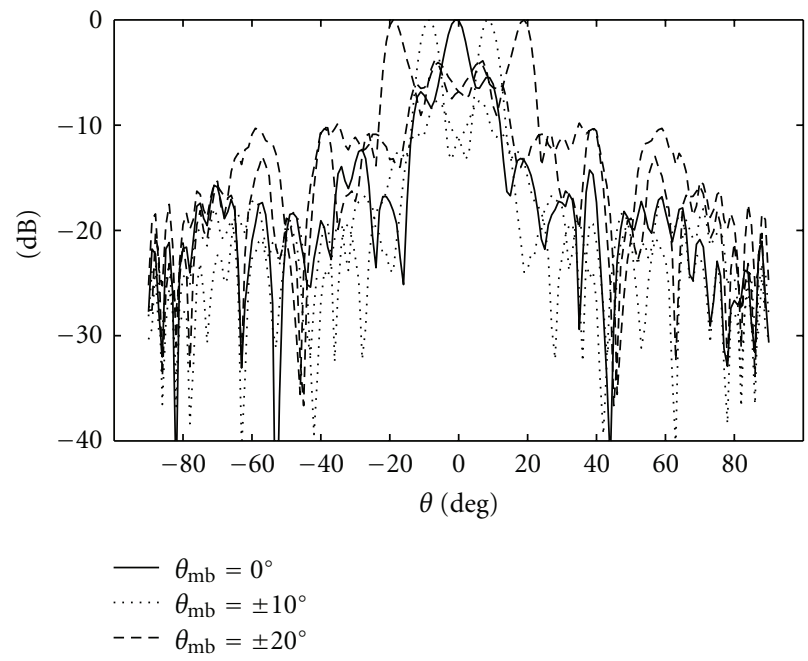

FIGURE 7: Normalized radiation patterns (copolar components on the $x-z$ plane) measured for different biasing voltage distributions.

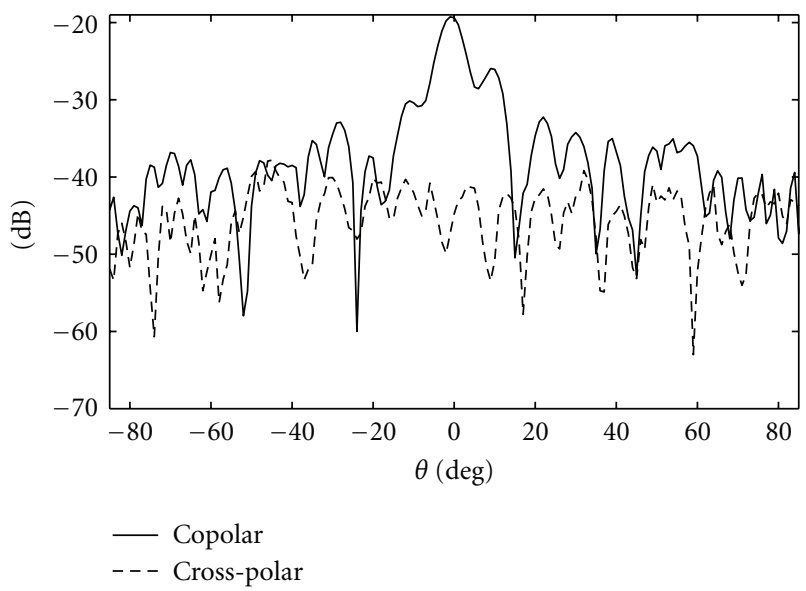

FIGURE 8: Measured radiation pattern (copolar and cross-polar components on $x-z$ plane) for a main beam $\theta_{\mathrm{mb}}=0^{\circ}$.

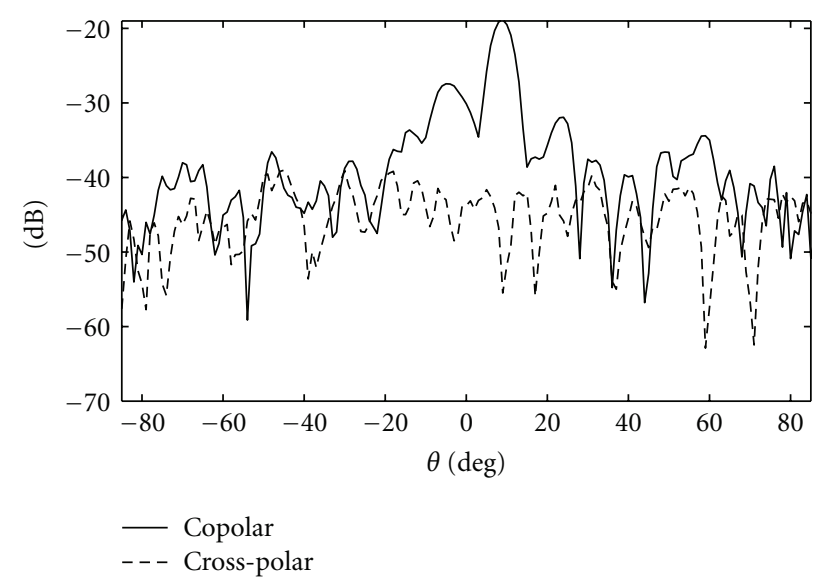

FIGURE 9: Measured radiation pattern (copolar and cross-polar components on $x-z$ plane) for a main beam $\theta_{\mathrm{mb}}=10^{\circ}$.

The reflectarray antenna can be easily reconfigured by a PC, which communicates with the ATmega1284P microcontroller through an USART interface. The microcontroller sends the desired voltage levels to the DACs array, which is properly connected to the varactor diodes.

The fabricated structure, illustrated in Figure 6, is validated by performing the measurement of the radiation pattern for different configurations of the bias voltages across the diodes. The considered voltage distributions are determined in order to scan the radiation pattern main beam $\theta_{\mathrm{mb}}$ from -20 to 20 degrees in the $x-z$ plane (Figure 4$)$. The required bias voltages levels are computed by a synthesis algorithm based on the iterative projection method [15].

A far-field measurement facility is adopted, which is placed into the anechoic chamber of the University of Calabria.

A double-ridged broadband horn, operating within the frequency range equal to $(1 \div 18) \mathrm{GHz}$, is adopted as probe at a far-field distance of $5.6 \mathrm{~m}$ from the reflectarray.

The measured copolar patterns on the $x-z$ plane, reported under Figure 7 , show the main beam $\theta_{\mathrm{mb}}$ effectively scanned along the assigned directions. A measurement frequency equal to $11.25 \mathrm{GHz}$ is assumed. As the aim of the realized prototype is to simply demonstrate the validity of the proposed scanning technique, a small number of elements is assumed on the $y-z$ plane, with no specific tuning, so the characterization of the radiation patterns on this plane has no significant validity and it is not reported.

As a further characterization, the cross-polar patterns on the $x-z$ plane are measured for three different main beam conditions, namely, $\theta_{\mathrm{mb}}=0^{\circ}, 10^{\circ}, 20^{\circ}$, and the comparison with the relative copolar components is reported in Figures 8, 9 , and 10. In all cases, a cross-polarization approximately below $-40 \mathrm{~dB}$ can be observed.

A loss analysis on the reflectarray is performed for a main beam in the broadside direction. The measured gain versus frequency is reported in Table 1 . In particular, at the central design frequency of $11.25 \mathrm{GHz}$, a gain value of $9.3 \mathrm{dBi}$ is obtained. At the same frequency, the maximum 


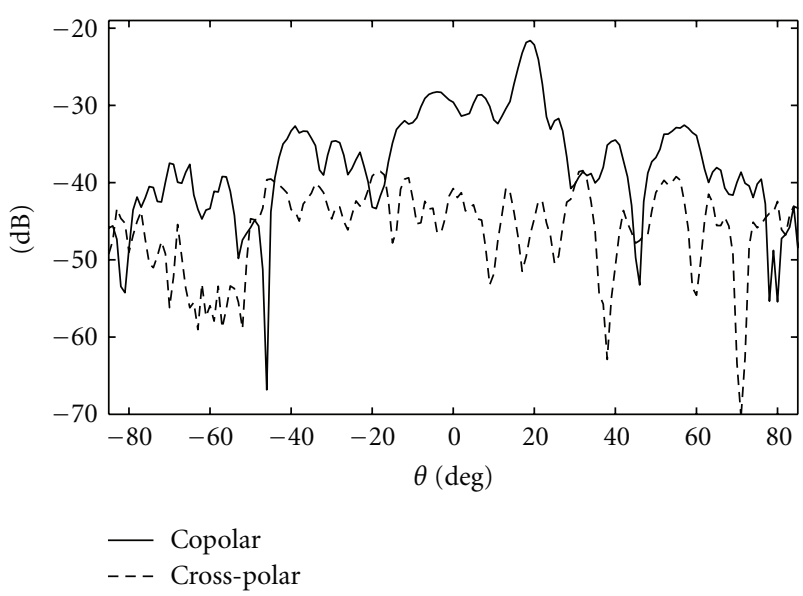

Figure 10: Measured radiation pattern (copolar and cross-polar components on $x-z$ plane) for a main beam $\theta_{\mathrm{mb}}=20^{\circ}$.

Table 1: Measured Gain versus Frequency (Broadside Configuration).

\begin{tabular}{lc}
\hline Frequency $(\mathrm{GHz})$ & Gain $(\mathrm{dB})$ \\
\hline 10.9 & 6.6 \\
11 & 8.1 \\
11.1 & 7 \\
11.15 & 7.8 \\
11.2 & 8.6 \\
11.25 & 9.3 \\
11.35 & 5.8 \\
11.5 & 6.6 \\
\hline
\end{tabular}

directivity for a reflectarray with an aperture area of $21.21 \lambda^{2}$ is calculated as $24.24 \mathrm{dBi}$. Using the measured gain, the antenna efficiency is estimated as $3.3 \%$. This small value is mainly due to the high spillover in the $y-z$ plane.

\section{Conclusion}

An aperture-coupled patch loaded by a single tunable varactor diode has been adopted for the implementation of a reconfigurable reflectarray prototype. The good phase agility associated to the designed reflectarray element has allowed the realization of a $3 \times 15$ reflectarray antenna with beamscanning capabilities within an angular range of about 40 degrees. The experimental tests on the fabricated antenna have demonstrated the validity of the implemented reflectarray configuration.

\section{References}

[1] D. M. Pozar and T. A. Metzler, "Analysis of a reflectarray antenna using microstrip patches of variable size," Electronics Letters, vol. 29, no. 8, pp. 657-658, 1993.

[2] J. Huang, "Microstrip reflectarray," in Proceedings of the IEEE International Symposium on Antennas and Propagation, pp. 612-615, Ontario, Canada, June 1991.
[3] J. A. Encinar and J. Agustín Zornoza, "Broadband design of three-layer printed reflectarrays," IEEE Transactions on Antennas and Propagation, vol. 51, no. 7, pp. 1662-1664, 2003.

[4] E. Carrasco, M. Barba, and J. A. Encinar, "Reflectarray element based on aperture-coupled patches with slots and lines of variable length," IEEE Transactions on Antennas and Propagation, vol. 55, no. 3, pp. 820-825, 2007.

[5] J. Huang and R. J. Pogorzelski, "A ka-band microstrip reflectarray with elements having variable rotation angles," IEEE Transactions on Antennas and Propagation, vol. 46, no. 5, pp. 650-656, 1998.

[6] M. E. Cooley, J. F. Walker, D. G. Gonzalez, and G. E. Pollon, "Novel reflectarray element with variable phase characteristics," IEE Proceedings, vol. 144, no. 2, pp. 149-151, 1997.

[7] S. V. Hum, M. Okoniewski, and R. J. Davies, "Realizing an electronically tunable reflectarray using varactor diode-tuned elements," IEEE Microwave and Wireless Components Letters, vol. 15, no. 6, pp. 422-424, 2005.

[8] M. Riel and J. J. Laurin, "Design of an electronically beam scanning reflectarray using aperture-coupled elements," IEEE Transactions on Antennas and Propagation, vol. 55, no. 5, pp. 1260-1266, 2007.

[9] F. Venneri, S. Costanzo, and G. Di Massa, "Reconfigurable aperture-coupled reflectarray element tuned by single varactor diode," Electronics Letters, vol. 48, no. 2, pp. 68-69, 2012.

[10] A. Moessinger, S. Dieter, W. Menzel, S. Mueller, and R. Jakoby, "Realization and characterization of a $77 \mathrm{GHz}$ reconfigurable liquid crystal reflectarray," in Proceedings of the 13th International Symposium on Antenna Technology and Applied Electromagnetics and the Canadian Radio Sciences Meeting (ANTEM/ URSI '09), February 2009.

[11] J. P. Gianvittorio and Y. Rahmat-Samii, "Reconfigurable patch antennas for steerable reflectarray applications," IEEE Transactions on Antennas and Propagation, vol. 54, no. 5, pp. 13881392, 2006.

[12] R. Sorrentino, R. V. Gatti, L. Marcaccioli, and B. Mencagli, "Electronic steerable mems antennas," in Proceedings of the European Conference on Antennas and Propagation (EuCAP '06), November 2006.

[13] J. Perruisseau-Carrier and A. K. Skrivervik, "Monolithic MEMS-based reflectarray cell digitally reconfigurable over a $360^{\circ}$ phase range," IEEE Antennas and Wireless Propagation Letters, vol. 7, pp. 138-141, 2008.

[14] O. Bayraktar, O. A. Civi, and T. Akin, "Beam switching reflectarray monolithically integrated with RF MEMS switches," IEEE Transactions on Antennas and Propagation, vol. 60, no. 2, part 2, Article ID 6056550, pp. 854-862, 2012.

[15] F. Venneri, S. Costanzo, G. Di Massa, and G. Angiulli, "An improved synthesis algorithm for reflectarrays design," IEEE Antennas and Wireless Propagation Letters, vol. 4, no. 1, pp. 258-261, 2005. 

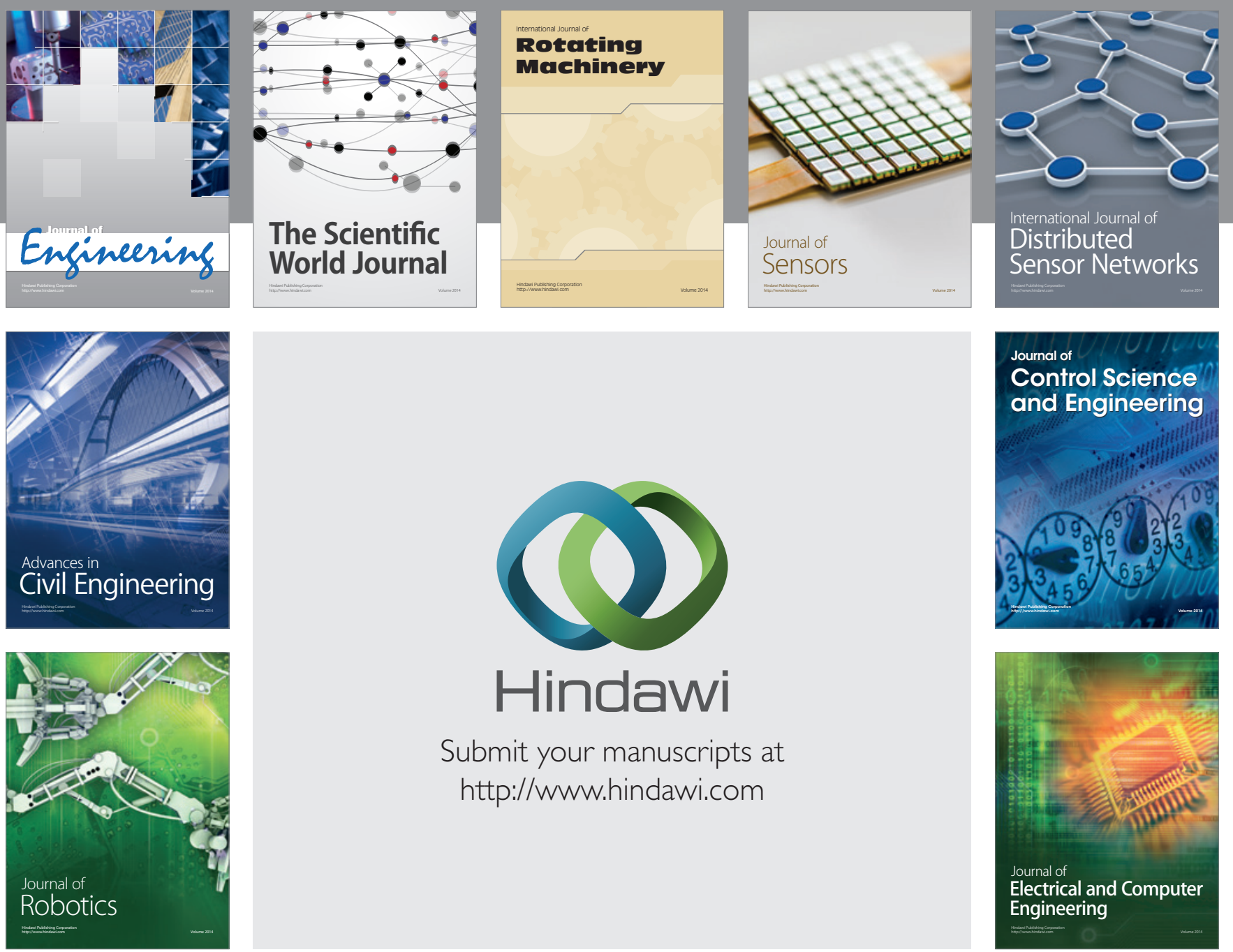

Submit your manuscripts at

http://www.hindawi.com
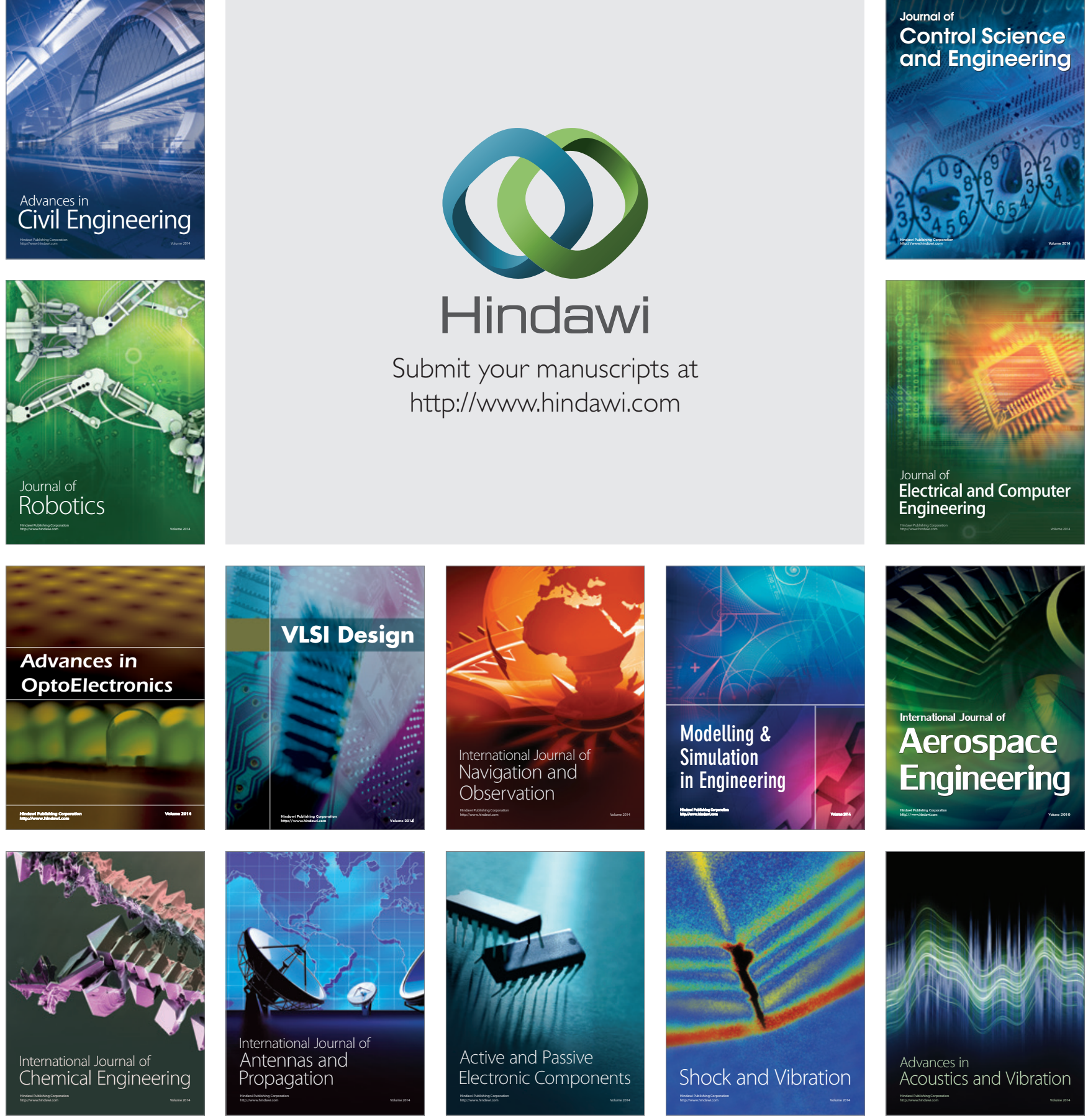